\title{
LA DISLEXIA COMO MANIFESTACIÓN DE NEURODIVERSIDAD
}

\author{
Dyslexia: neurodiversity manifesting
}

\author{
Yenny Alejandra Beltrán Rodríguez \\ yabeltranr@correo.udistrital.edu.co \\ Universidad Distrital Francisco José de Caldas \\ Licenciatura en Biología \\ Colombia \\ Gabriel Gutiérrez Ospina \\ gabo@biomedicas.unam.mx \\ Universidad Nacional Autónoma de México \\ Instituto de Investigaciones Biomédicas \\ México
}

\section{RESUMEN}

La habilidad lectora está representada por una curva de distribución normal en la que el fenotipo denominado disléxico constituye aproximadamente el 17\%. Esta distribución y frecuencia hacen de este fenotipo una expresión alternativa de la normalidad lectora en el ser humano. En este trabajo se propone un modelo que mira a la dislexia como una manifestación de neurodiversidad, superando las ideas que la presumen como una neuropatología. En él se sugiere que las variantes disléxicas son alternativas fenotípicas que permiten a los individuos desarrollar habilidades cognitivas novedosas y adaptativas. Para este fin, se analizó el marco conceptual y experimental de 143 artículos científicos obtenidos de las bases de datos ScienceDirect, NCBI y PubMed, seleccionados bajo los términos de búsqueda "dislexia" y "lectura", relacionados con su neurobiología, diagnóstico, características y neurodiversidad. La inclusión adicional de datos neurofisiológicos y neuroanatómicos, representados por modelos a lo largo del trabajo, permitió ilustrar la diversidad de las organizaciones cerebrales que ratifican la idea de que los "cerebros disléxicos" no representan un rasgo fenotípico inadaptado, sino más bien un arreglo de organizaciones cerebrales que proporcionan posibilidades cognitivas diversas. En consecuencia, se construyó un modelo neurobiológico teórico para lectura y dislexia que procura predecir las múltiples posibilidades de procesamiento de la información para ambos fenotipos. Esta teorización condujo a proponer un nuevo concepto para referirse a las variantes "disléxicas" del fenotipo humano, la holodisnomia, término que aspira a sustituir el término dislexia y que atiende a la capacidad de los individuos que lo exhiben de procesar la información de manera global (holo-), así como desarrollar una estructura de pensamiento que diverge positivamente de la norma (-disnomia).

\section{PALABRAS CLAVE:}

Neurobiología, dislexia, lectura, neurodiversidad

Cite este artículo como: Beltrán, Y., \& Gutiérrez, G. (2019). La dislexia como manifestación de neurodiversidad. 


\section{INTRODUCCIÓN}

Un rasgo fenotípico sobresaliente del ser humano es su capacidad para adquirir información por medio de la lectura. Esta capacidad varía interindividualmente de manera significativa, por lo que no existe un solo fenotipo lector en el grueso de la población humana. Si bien algunas voces han considerado a la dislexia como un fenotipo categórico incapacitante/inadaptado, es menester recordar que existe "una gama de dislexias", como de todos los fenotipos lectores, cada uno de ellos exhibiendo cualidades circunstancialmente ventajosas en función de los contextos en los que se desempeñen los individuos que los manifiesten. Esto sugiere que los fenotipos lectores emergen de manera natural en cada población de individuos dada, como el resultado de respuestas contingentes del cerebro en desarrollo a retos circunstanciales que se presentan durante la vida prenatal y postnatal, por lo que considerarlas patologías resulta cuando menos insensato. Al respecto, conjeturamos que las variantes fenotípicas "disléxicas" constituyen alternativas que permiten a aquellos individuos que las muestran el desarrollo de habilidades cognitivas altamente adaptativas. Con este fin se realizó una revisión, análisis y propuesta teórica sobre la neuroanatomía y neurofisiología de la dislexia y del lenguaje dirigida a despatologizar a estas variantes lingüísticas a través de la construcción de un nuevo modelo neurobiológico que procura predecir las múltiples posibilidades de procesamiento de la información para los fenotipos eu-lector y "disléxico". Este abordaje, reflejado en el modelo actualizado de las rutas neurales empleadas en procesos de lectura e involucradas en los rasgos fenotípicos exhibidos por la población con "dislexia" condujo a la creación de un nuevo concepto que abarca el amplio espectro de rasgos fenotípicos adaptativos de las "personas disléxicas". Este término, la holodisnomia, remarca las cualidades que las "personas disléxicas" poseen: procesamiento holístico de la información acompañada de un pensamiento divergente (i.e., creativo).

\section{MÉTODO}

Se analizaron 143 artículos científicos obtenidos de las bases de datos ScienceDirect, PubMed y NCBI bajo los términos de búsqueda "dislexia" y "lectura". Se seleccionaron solo aquellas publicaciones que contaran con datos neurofisiológicos y neuroanatómicos que posteriormente pudiesen ser incorporados al modelo renovado de holodisnomia.

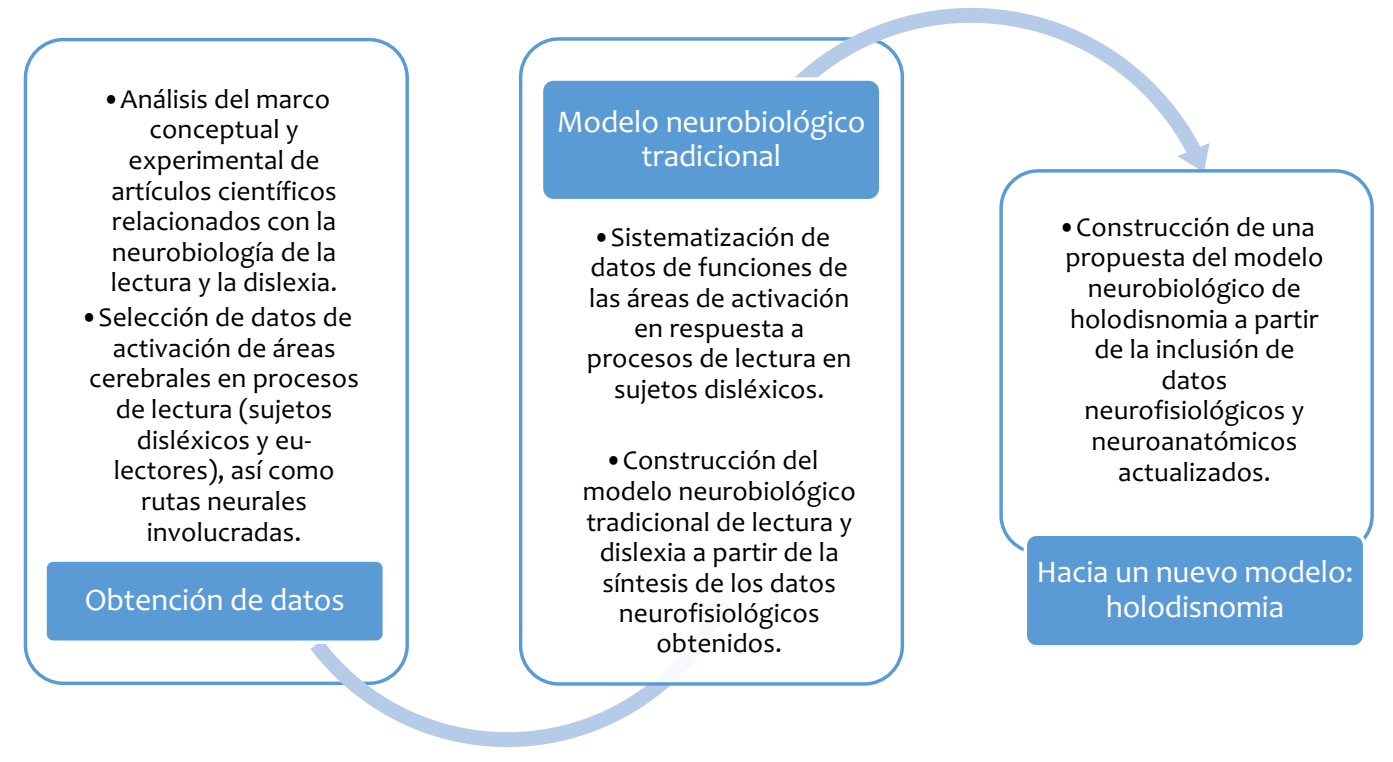




\section{RESULTADOS Y DISCUSIÓN}

\section{Modelo neurobiológico tradicional para lectura y "dislexia"}

Estudios de imagenología funcional sugieren que en individuos eu-lectores, la lectura está sostenida por una estructura neural compuesta por cuatro regiones cerebrales ubicadas en el hemisferio izquierdo. Estas regiones son la occipito-temporal ventral (OT; área visual encargada de identificar la forma de las palabras), la circunvolución frontal inferior (IFG), la circunvolución precentral dorsal ( $\mathrm{PrC}$ ) y la corteza parieto-temporal (TP), la última conformada por el lóbulo parietal inferior dorsal (IPL) y la circunvolución temporal superior (STG) (Richlan, 2014; Figura 1). Las regiones mencionadas se conectan entre sí a través de dos haces referidos como dorsal y ventral, constituidos, respectivamente, por los fascículos longitudinal superior y el arqueado, y los fascículos longitudinal inferior fronto-occipital y el uncinado. En su conjunto, se cree que el haz dorsal transmite información fonológica y articulatoria, mientras que el haz ventral se encarga de transmitir información semántica (Cuadro 2). De esta forma, las vías dorsales parecen más involucradas en la conversión grafofonológica mientras que las ventrales permiten el acceso léxico-semántico (Bertolotti et al., 2017; Jobard et al., 2003; ver también Hickok \& Poeppel, 2004; Saur et al., 2008).

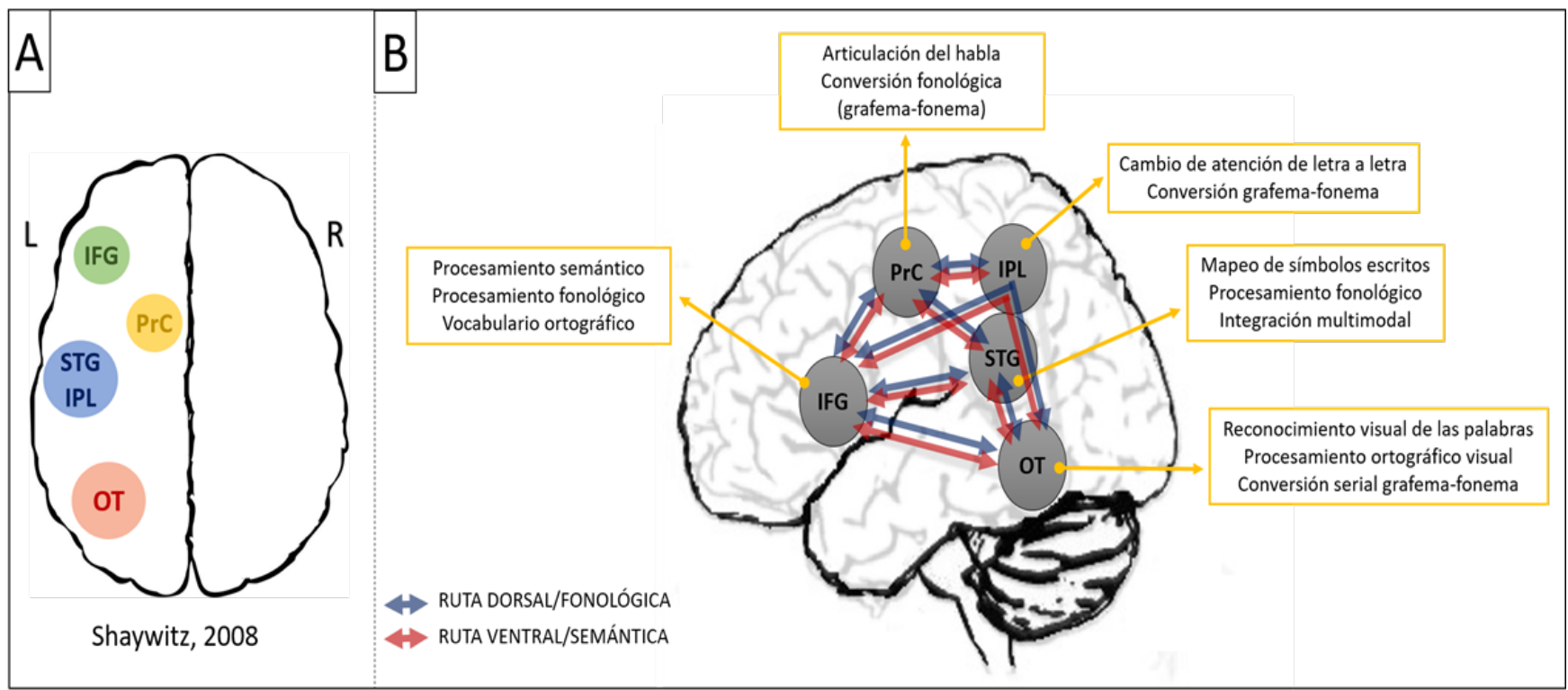

Figura 1. Modelo de la circuitería neural que soporta a la lectura. A) Áreas cerebrales involucradas en la lectura. IFG: circunvolución frontal inferior; PrC: circunvolución precentral; IPL: lóbulo parietal inferior; STG: circunvolución temporal superior; OT: corteza occipitotemporal. B) Circuitos que interconectan a las distintas áreas que subyacen a la lectura. Las flechas azules corresponden al haz dorsal. Las rojas al haz ventral. Se postula que son multidireccionales. Modificado de Richlan (2014), funciones de las áreas (Peterson \& Pennington, 2012), rutas (Perrone-Bertolotti et al., 2017). 
Cuadro 2. Rutas dorsal y ventral para el reconocimiento visual de las palabras en procesos de lectura, según Bertolotti et al. (2017).

\section{RUTA DORSAL}

PROCESAMIENTO FONOLÓGICO

- Lóbulo parietal inferior (IPL): conversión grafo-fonémica.

- Circunvolución supramarginal: conversión grafo-fonémica.

- Circunvolución temporal superior (STG): reconocimiento y selección de fonemas.

- Circunvolución frontal inferior (IFG): activación de códigos de fonemas.

- Interacción de la corteza occipito-temporal ventral (OT)/VWFA con IFG: codificación de entradas visuales de letras a sonidos o fonemas (conversión grafo-fonémica).

Conexiones anatómicas: Saur et al., 2008; Friederici y Gierhan, 2013; Kellmeyer et al., 2013 en Bertolotti et al., 2017.

Conexión temporal-parietal-frontal: fascículo longitudinal superior. Conexión fronto-temporal: fascículo arqueado.
RUTA VENTRAL

PROCESAMIENTO LÉXICO-SEMÁNTICO

- Activación de la región occipito-temporal.

- Área temporal medial e inferior: ITG, MTG: acceso y recuperación de representaciones léxicas.

- Circunvolución temporal anterior: procesamiento semántico.

- Giro angular: memoria semántica.

- Parte anterior del IFG: recuperación semántica y procesos semánticos de más alto nivel.

Conexiones anatómicas: Saur et al., 2008; Friederici y Gierhan, 2013; Kellmeyer et al., 2013 en Bertolotti et al., 2017.

Dependen del uncinado y del fascículo inferior longitudinal fronto-occipital.

Con base en lo expresado con antelación, para el caso de los individuos que manifiestan el "fenotipo disléxico", se presume que las regiones OT, IFG, PrC, IPL y STG, así como los haces dorsal y ventral (o sus fascículos) presentarían alteraciones anatomo-funcionales que tendrían como consecuencia la manifestación de "dificultades" para lograr la conversión grafo-fonológica y la recuperación del significado de las palabras. En apoyo a esta posibilidad, se han generado datos anatómicos que muestran una disminución del volumen de materia gris en las regiones STG, IPL, IFG, OT, circunvolución fusiforme izquierda (FFG), surco temporal superior (STS) y cerebelo (C) (Linkersdörfer et al., 2012; Brown et al., 2001; Eckert et al., 2016; Richlan et al., 2013). Funcionalmente, Paulesu et al. (2001) reportaron una disminución del área de activación en la STG y en la OT. De hecho, la OT izquierda parece no reconocer al mismo tiempo todas las letras que constituyen una palabra (Zoccolotti et al., 2005 citado por Dehaene, 2014).

Bajo la misma óptica "patologizante", la dislexia también se ha asociado con actividad reducida en regiones temporo-parietales (TP) relacionadas con procesamiento fonológico (Richlan et al., 2011). Estudios enfocados a estudiar la integridad de conectividad cortical subyacente a la lectura documentan que el fascículo longitudinal superior del hemisferio izquierdo, el fascículo arqueado y el fascículo fronto-occipital inferior (Vandermosten et al., 2012) están reducidos de tamaño en sujetos que muestran "fenotipos disléxicos" (Klingberg et al., 2000; Beaulieu et al., 2005; Deutsch et al., 2005; Silani et al., 2005; Niogi \& McCandliss, 2006 citados en Dehaene, 2014; Kershner, 2016), lo que aparentemente sugiere que la región temporal izquierda está desconectada de otras regiones cerebrales, causando una importante alteración en el flujo de información a la IFG (Dehaene, 2014; Yeatman et al., 2012). Finalmente, la baja activación en PT y OT (Pugh et al., 2000; Richlan et al., 2009) parece afectar el reconocimiento fluido de las palabras, mientras que la sobreactivación en los sistemas anteriores se relaciona con la utilización de recursos fono-articulatorios para decodificar palabras desconocidas o pseudopalabras (Pearson, 2017).

En resumen, se ha propuesto un esquema general de activación del "cerebro disléxico" en respuesta a tareas de lectura (Simos et al., 2007; Shaywitz \& Shaywitz, 2008) representado por la subactivación de las regiones posteriores del hemisferio izquierdo (PT y OT), y sobreactivación de la PrC del hemisferio izquierdo, la IFG de ambos hemisferios, así como la OT del hemisferio derecho (Figura 2). Aunque el arreglo morfo-funcional se juzga como deficiente e inadaptado, es importante notar que, en el cerebro de sujetos con "fenotipo disléxico" los núcleos lectores se activan en ambos hemisferios cerebrales, es decir, la lectura es bilateral. Estos cambios sugieren más bien que la organización morfofuncional del "cerebro disléxico" representa una alternativa que resuelve los retos a los que se enfrenta generando estrategias adaptativas distintas debido, en parte, a sus propiedades sensoriomotoras y perceptuales divergentes. 


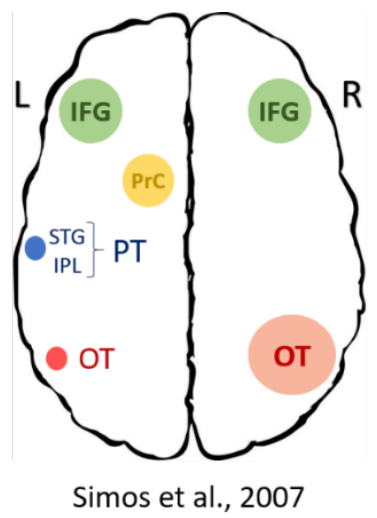

Figura 2. Áreas de activación en procesos de lectura en el cerebro de individuos que presentan un "fenotipo disléxico". Las diferencias en los patrones de activación se encuentran representadas por el tamaño de los círculos. Nótese la bilateralidad de la representación de la lectura. L: Hemisferio izquierdo; R: Hemisferio derecho.

\section{Compensación y diversidad morfofuncional en el "fenotipo disléxico"}

La identificación del "fenotipo disléxico" por parte del personal médico y/o psicológico capacitado pareciera ser sencilla y categórica. Nada más alejado de la realidad. La heterogeneidad de las manifestaciones cognitivas de sujetos que son identificados como "disléxicos", y de sus correlatos neuronales, es tan amplia como aquella observada entre los fenotipos eu-lectores, la segunda, considerada parte de la variación natural de la especie. De hecho, y en favor de esta última aseveración, baste recordar que la frecuencia de la dislexia en la población llega a alcanzar el 17\% (APA, 2013; Barbiero et al., 2012; Zakopoulou et al. 2011; Shaywitz et al., 1992), lo que la transforma de una anomalía en un fenotipo frecuente cobijado por la curva de normalidad de la diversidad neural humana. Además, "el fenotipo disléxico", como ya se subrayó, no es categórico, sino que está representado por matices. Como ejemplo, baste un botón. Estudios de morfología funcional han reportado la existencia de un patrón global de activación cortical y subcortical durante la lectura que se presume común a los sujetos "disléxicos", pero que difiere del observado en los sujetos control. Este patrón se caracteriza por la activación bilateral de los ganglios basales (Bg), la IFG y la ínsula (I) (aunque ver Bach et al., 2010), unilateral derecho de la OT y C y unilateral izquierdo de la PrC, el tálamo (Th) y la circunvolución post central (PoC) (Figura 3; Shaywitz et al., 2002; Simos et al., 2007; Shaywitz y Shaywitz, 2008; Ingvar et al., 2002; Bach et al., 2010; Hancock et al., 2017; Hoeft et al., 2007; Kronbichler et al., 2006; Maisog et al., 2008; Richlan et al., 2009, 2011; Wimmer et al., 2010; Meyler et al., 2008). Es de resaltar que algunas de las regiones que forman parte de este patrón no se relacionan primariamente con el proceso de lectura en individuos eu-lectores (aunque ver Paulesu et al., 1993; McNorgan et al., 2011; Hancock et al., 2017), lo que sugiere que el cerebro en los sujetos "disléxicos" representa una organización alternativa que le permite el desarrollo de estrategias fonológicas y/o articulatorias compensatorias. 


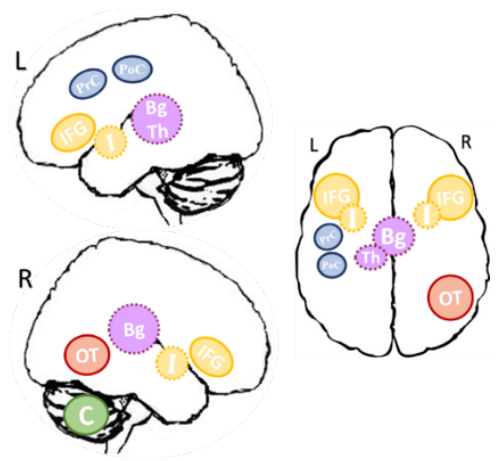

Figura 3. Patrón de activación global cortical y subcortical durante la lectura que se presume característico de sujetos "disléxicos". PrC: circunvolución precentral; PoC: circunvolución postcentral; IFG: circunvolución frontal inferior; I: ínsula; Bg: ganglios basales; Th: tálamo; OT: región occipitotemporal; C: cerebelo.

Si bien los patrones de activación cortical cerebral descritos se piensan característicos de la dislexia, en realidad constituyen la base sobre la cual se construye la diversidad morfo-funcional del "cerebro disléxico". Esta diversidad se manifiesta al estudiar los patrones de activación cortical cerebral consecutivos a una intervención terapéutica (ie., entrenamiento en habilidades fonológicas, procesamiento auditivo y lenguaje oral; Fig. 4), instrumentada con el propósito de mejorar su desempeño en la lectura. Independientemente de que los individuos mejoren la precisión de sus habilidades de lectura como resultado de la intervención, los patrones de activación difieren entre los individuos, siendo estos significativamente más divergentes entre los individuos que responden a la intervención. De hecho, estos últimos patrones de activación no corresponden a aquellos observados en individuos eu-lectores, observación que apoya que la organización cerebral en los "individuos disléxicos" en verdad representa una respuesta contingente, una alternativa funcional y adaptativa para ellos, además de reflejar una diversidad de arreglos morfofuncionales cerebrales que permiten la expresión poblacional de un espectro fenotípico "disléxico".

Modelo de compensación con intervención

A) PPR: Problemas persistentes para leer post-intervención
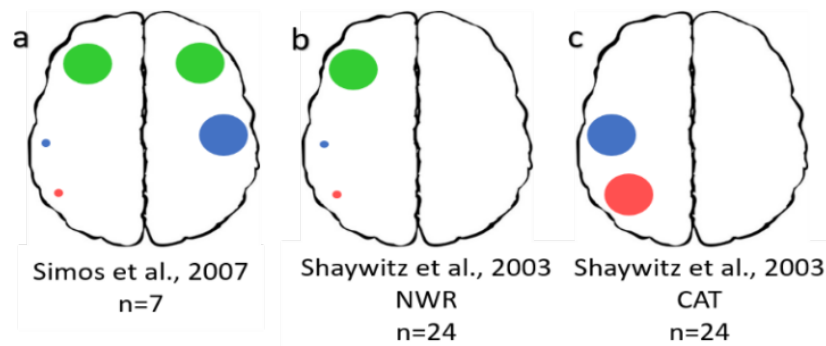

B) AIR: Mejora en habilidades de lectura post-intervención
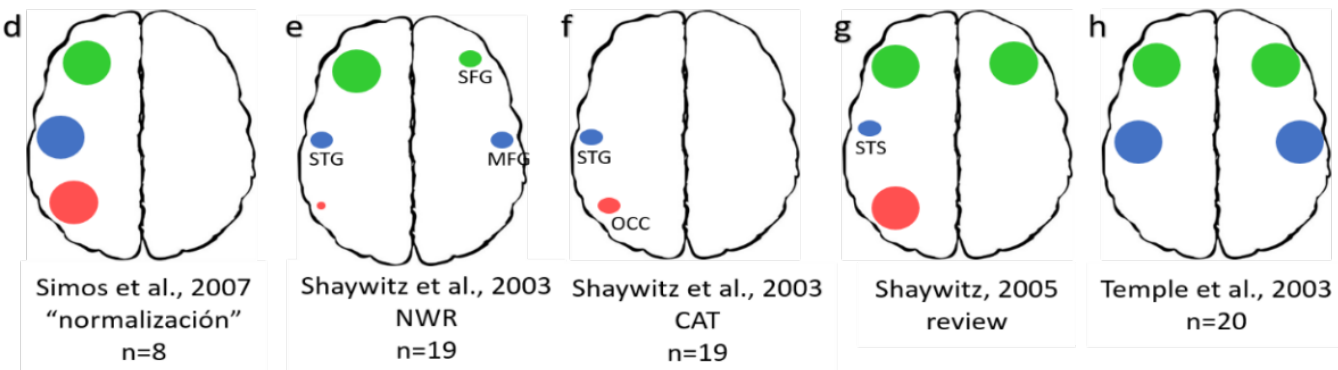

Figura 4. Patrones de activación cortical cerebral posteriores a una intervención de entrenamiento en habilidades fonológicas, procesamiento auditivo y lenguaje oral en sujetos que muestran problemas persistentes para leer (A) y en sujetos que mejoran sus habilidades de lectura (B). Convenciones de color: 
verde, IFG; azul, PT; rojo, OT. Nótese que la diversidad interindividual del fenotipo disléxico es mayor en sujetos que responden positivamente a la intervención, lo que sugiere que el arreglo morfofuncional subyacente es adaptativo y funcional.

\section{"Cerebro holodisnómico": Expresión alternativa de neurodiversidad}

Se espera que, con los datos comentados en la sección precedente, quede claro para el lector que la organización morfo-funcional cerebral en los "fenotipos disléxicos" constituye, en realidad, una solución diferente con posibilidades adaptativas y de funcionalidad plena, en contextos acordes con su diseño. Si esto fuera así, se esperaría observar "ventajas" o "fortalezas" cognitivo-conductuales en individuos calificados como disléxicos al comparar su desempeño con individuos eu-lectores, en tareas diferentes a la lectura, tal y como se observa en los procesos de plasticidad adaptativa evaluados en individuos invidentes o con pérdidas auditivas (Martínez-Méndez et al., 2013). Como ejemplo de ello, se ha mostrado que modificaciones en el proceso de integración de la información visual periférica en los “individuos disléxicos” les permite llevar a cabo tareas de análisis global de las imágenes y de identificación de anomalías presentes en el contexto visual, evaluado de forma más eficaz que el anotado para individuos eu-lectores (Schneps et al., 2007). Otras fortalezas cognitivo conductuales asociadas con el "fenotipo disléxico" se presentan en el Cuadro 3. Al revisar el cuadro es posible observar que las organizaciones morfofuncionales de los cerebros en "individuos disléxicos" los capacitan, por encima de los fenotipos eu-lectores, para integrar sensorio-perceptualmente, de manera holística y generalista, la información circundante, proveyéndoles de la posibilidad de generar respuestas conductuales contingentes, divergentes y creativas, por lo que definir a este fenotipo como "holodisnómico" despatologiza a la variante pues hace referencia al amplio espectro de manifestaciones conductuales exhibidas por los individuos que la poseen. Es justo articular, por tanto, que las fortalezas del fenotipo holodisnómico no se presentan "a pesar de sus dificultades en la ejecución de tareas de lectura y escritura", sino que reflejan que sus cerebros están configurados estructural y funcionalmente, de tal manera que exhiben otras facultades de procesamiento de información (Eide \& Eide, 2012). Estos hallazgos están en línea con la idea de que la dislexia (de ahora en más holodisnomia), no representa un fenotipo discapacitado, sino que implica a una pléyade de variantes funcionales cobijados por la curva de normalidad estadística (Pennington, 2006). Es importante mencionar que las fortalezas cognitivo-conductuales observadas en los individuos holodisnómicos van más allá de descripciones fenoménicas bajo condiciones relativamente controladas. En verdad, estas poseen un correlato neural. Sirva de ejemplo que la capacidad de los sujetos holodisnómicos para atender el aspecto global de las figuras se correlaciona con la activación de la corteza temporo-occipital del hemisferio derecho (Fink et al., 1996). Elementos críticos de la creatividad artística visual como la atención global y el pensamiento viso-espacial observados en individuos holodisnómicos están mediados por el lóbulo parietal derecho (Heilman \& Acosta, 2013). De forma similar, se ha propuesto que los lóbulos frontales en individuos holodisnómicos podrían ser críticos para el pensamiento divergente (Heilman \& Acosta, 2013), y que el hemisferio derecho modula su capacidad de producción artística, así como la atención global (Cohn \& Neumann, 1997). Finalmente, se ha considerado que una "disfunción del hemisferio dominante" puede dar lugar a la desinhibición del lóbulo parietal "no dominante", resultando en un desenmascaramiento de talento artístico oculto (Kapur, 1996; entrecomillado usado por los autores del presente ensayo). Con ello, la habilidad facilitada de los individuos holodisnómicos para resolver tareas en las que se requiere romper las convencionalidades podría explicar sus "dificultades" para adquirir las reglas convencionales del lenguaje y la gramática específica (Bigozzi et al., 2016; entrecomillado usado por los autores del presente ensayo)

Cuadro 3. Características sensoperceptuales y cognitivas fortalecidas observadas en sujetos holodisnómicos.

\begin{tabular}{|l|l|}
\hline VENTAJAS & REFERENCIA BIBLIOGRÁFICA \\
\hline Habilidades viso-espaciales: tareas de completar figuras, ensamblaje de & Schneps et al., 2007; Sladen, 1972; Rigel, 1974; \\
objetos y diseño de bloques, análisis de imagen, comparación visual & Rack, 1981; Olagboyega, 2008; Diehl et al., 2014; \\
(percepción de anomalías o inconsistencias) y discriminación de figuras & Schneps, 2015; von Karolyi, 2001; von Karolyi et \\
\hline
\end{tabular}




\begin{tabular}{|c|c|}
\hline $\begin{array}{l}\text { posibles e imposibles: gracias a una visión periférica favorecida. } \\
\text { Procesamiento visual holístico. }\end{array}$ & al., 2003; von Karolyi \& Winner, 2004 \\
\hline Creatividad (artística o literaria). & $\begin{array}{l}\text { Rack, 1981; Olagboyega, 2008; Pearson, 2017; } \\
\text { Kiziewicz \& Biggs, 2007; Chakravarty, } 2009\end{array}$ \\
\hline $\begin{array}{l}\text { Imaginería visual: pensamiento espacial multidimensional (preferencia } \\
\text { para el pensamiento en tres dimensiones); tareas de rotación mental: } \\
\text { capacidad de identificar elementos (letras, figuras o imágenes) } \\
\text { presentados con diferentes orientaciones. }\end{array}$ & $\begin{array}{l}\text { Olagboyega, 2008; Schneps, 2015; Eide \& Eide, } \\
2012\end{array}$ \\
\hline $\begin{array}{l}\text { Resolución de problemas (creatividad): encontrar formas alternativas } \\
\text { para resolver; pensamiento divergente y creativo. }\end{array}$ & $\begin{array}{l}\text { Olagboyega, 2008; Pearson, 2017; Heilman \& } \\
\text { Acosta, } 2013\end{array}$ \\
\hline $\begin{array}{l}\text { Tendencia a prestar atención a muchas actividades concurrentes } \\
\text { (atención a múltiples entradas auditivas al mismo tiempo). }\end{array}$ & Geiger \& Lettvin, 1987 \\
\hline Persistencia (hábitos creados por el ejercicio prolongado). & Rawson, 1978 \\
\hline $\begin{array}{l}\text { Visión periférica optimizada para un procesamiento rápido en regiones } \\
\text { amplias (percepción de edificaciones y escenas). } \\
\text { Lectura favorecida en la periferia. }\end{array}$ & $\begin{array}{l}\text { Schneps et al, 2007; Schneps, 2011; Geiger \& } \\
\text { Lettvin, } 1987\end{array}$ \\
\hline $\begin{array}{l}\text { Aprendizaje espacial implícito (i.e., por exposición repetida a un espacio } \\
\text { que se hace familiar). }\end{array}$ & Howard et al., 2006 \\
\hline $\begin{array}{l}\text { Comprensión de conceptos gracias a habilidades de asociación de } \\
\text { significados. }\end{array}$ & Pearson, 2017 \\
\hline Memoria de imágenes (memoria visual). & Swanson, 1984; Hedenius et al., 2013 \\
\hline $\begin{array}{l}\text { Percepción de relaciones como analogías, metáforas, paradojas, } \\
\text { similitudes, diferencias, implicaciones, vacíos y desequilibrios. }\end{array}$ & Eide \& Eide, 2012 \\
\hline Habilidad para recordar experiencias personales importantes. & Eide \& Eide, 2012 \\
\hline $\begin{array}{l}\text { Habilidad para percibir y tomar ventaja de patrones sutiles en sistemas } \\
\text { complejos y constantemente cambiantes o conjuntos de datos. }\end{array}$ & Eide \& Eide, 2012 \\
\hline Producción artística. & Cohn \& Neumann, 1997; Pearson, 2017 \\
\hline Imaginación. & $\begin{array}{l}\text { Pearson, 2017; Cooper, 2009; Kiziewicz \& Biggs, } \\
2007\end{array}$ \\
\hline Procesamiento holístico de la información (vs. procesamiento secuencial). & Cooper, 2009 \\
\hline Reproducción de formas visuales complejas desconocidas. & Swanson, 1984 \\
\hline Encontrar vías alternativas para procesar la información. & Cockcroft \& Hartgill, 2004 \\
\hline Pensamiento divergente. & Bigozzi et al., 2016 \\
\hline
\end{tabular}

\section{Los "cerebros holodisnómicos": alternativas morfofuncionales}

En el modelo tradicional, las áreas corticales cerebrales que se presume subyacen a la lectura se reducen a las IFG, OT, PrC, STG e IPL del hemisferio izquierdo. Este modelo, sin embargo, ha ayudado a promover una visión "patologizada" de la holodisnomia, pues las áreas en él consideradas no reflejan la organización observada en el cerebro de los individuos holodisnómicos cuando estos ejecutan tareas de lectura. La revisión de la literatura contemporánea sobre el tema, tanto en individuos eu-lectores como en aquellos con fenotipo holodisnómico, brinda un panorama distinto, pues ahora se presume que la lectura requiere de la asociación funcional de 36 áreas cerebrales encargadas de llevar a cabo operaciones de procesamiento visual, memoria de trabajo, semántica, procesamiento auditivo, articulación del habla, imaginería visual y auditiva (Figura 5; Cuadro 6, anexo A; ver también Price, 2012). Cabe destacar que dentro de este conjunto de áreas existen aquellas que se consideraban exclusivas de la holodisnomia en referencia a la lectura (Figura 5), y que ahora han sido identificadas en los individuos eu-lectores. De hecho, algunas de ellas fueron primero observadas en individuos holodisnómicos (e.g., activación bilateral de Bg, IFG e I). En este nuevo modelo se propone que la integración 
de la información no ocurre de manera secuencial en una dirección dada, sino que resulta de la actuación concertada de áreas activadas concurrentemente e interactuando de forma flexible y dinámica dependiendo de las demandas de la tarea en ambos grupos de fenotipos. Así, el modelo predice la posibilidad de que se generen rutas alternativas de procesamiento en todos los individuos. Estas observaciones, nuevamente, permiten fortalecer el argumento de que las configuraciones morfofuncionales cerebrales observadas en los individuos holodisnómicos representan variaciones genuinas de la diversidad de la norma con posibilidades de adaptación y funcionamiento plenos.

Así, el modelo destaca la amplia distribución de los procesos de lectura a nivel cerebral. Los símbolos escritos serían procesados por el sistema visual y posteriormente seguirían rutas de procesamiento fonológico y semántico. Además, al tiempo se activan mecanismos de memoria de trabajo verbal, imaginería visual y auditiva, dependiendo de los requerimientos operativos. Las salidas motoras y articulatorias se asocian con estrategias de subvocalización empleadas por los sujetos de fenotipo holodisnómico y, a su vez, constituyen entradas sensoriales que podrían seguir la ruta de procesamiento auditivo. Esto evidencia una vez más las diversas posibilidades de procesamiento informático para el fenotipo holodisnómico teniendo en cuenta los mecanismos de plasticidad subyacente. Así mismo, destaca la participación moduladora del tálamo al regular la actividad cortical en respuesta a la lectura. La activación dinámica y flexible de las áreas (Figura 5) podría explicarse por el marcado solapamiento funcional que, por un lado, abre la posibilidad de que se generen rutas alternativas de procesamiento de información y, por el otro, permite estrategias de reorganización funcional como las exhibidas por el fenotipo holodisnómico, dado que un área determinada podría alternar entre diversas funciones. Esto probablemente se relaciona con la obtención de resultados de activación cerebral "inconsistentes" en sujetos eu-lectores y holodisnómicos, que posiblemente sean producto de la diversidad morfo-funcional exhibida por ambos grupos de individuos, reflejando más bien la naturaleza dinámica y diversa del modelo de lectura, en lugar de resultados conflictivos. Con base en lo anterior, uno podría esperar adaptaciones funcionales en los individuos holodisnómicos. En apoyo a esto, y a manera de ejemplo, la activación significativa de la región OT del hemisferio derecho se relaciona con la capacidad de atender al aspecto global de los estímulos presentados, y la activación bilateral de la región IFG se correlaciona con procesos de creatividad y pensamiento divergente. Ambos elementos facultan al sujeto holodisnómico para generar alternativas conductuales con respecto al sujeto eu-lector, situación que podría evaluarse en futuros estudios.

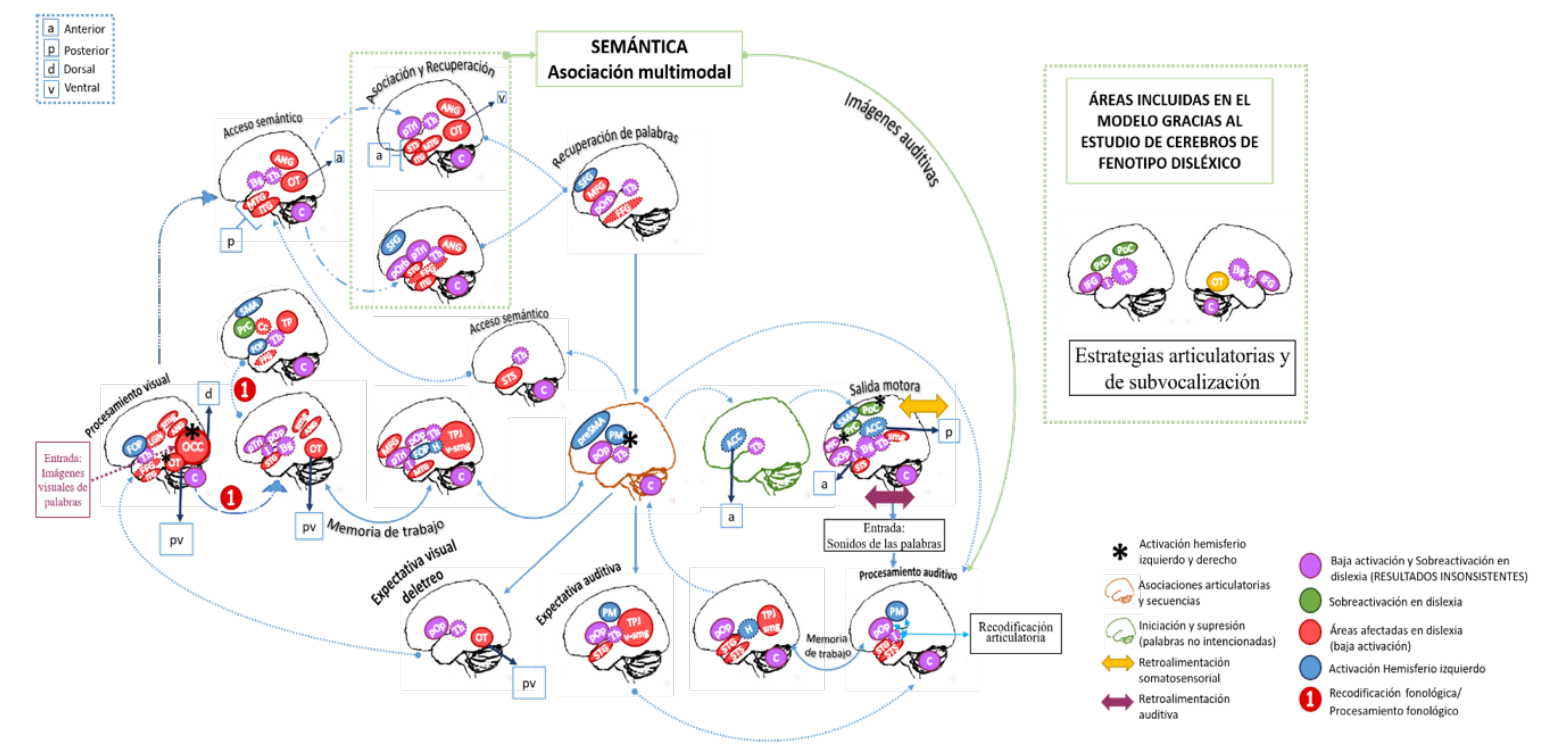

Figura 5. Modelo anatómico y funcional actualizado de la lectura (ver texto). 


\section{CONCLUSIÓN}

En suma, históricamente la dislexia (ahora holodisnomia) ha sido considerada como un trastorno neurológico derivado de un proceso de desarrollo ontogenético deficiente, que afecta principalmente a la esfera cognitiva relacionada con la lectura. Durante el desarrollo, sin embargo, el cerebro se ensambla dinámicamente a través de procesos auto-organizativos, respondiendo de manera contingente a los retos impuestos por el ambiente gestacional en muchas ocasiones estresante. Las respuestas del cerebro en desarrollo, reflejadas en su organización morfofuncional, son el resultado de "negociaciones" entre las distintas regiones que lo forman (i.e., ontogenia regulatoria; Adolph, 1968) y que conducen a "la mejor solución posible", considerando los eventos fortuitos que el cerebro afronta en distintos momentos durante la vida prenatal. De esta forma, la revisión cualitativa de la literatura desde una perspectiva incluyente (i.e., neurodiversa) y no patologizada, indica que la holodisnomia representa un fenotipo alternativo a la relativa norma fenotípica del cerebro humano. El individuo holodisnómico no solo despliega, como se ha hecho creer, un conjunto de características cognitivas divergentes que aparentemente dificultan, bajo las formas actuales de enseñanza y estructura social, la adquisición y ejecución de la lectura (situación que por cierto tiende a resolverse en menor o mayor grado a lo largo de la vida), sino que muestra habilidades sobresalientes relativas al procesamiento e integración de la información sensorial relacionada con la expresión creativa, que le facilitan fabricar una visión y concepción general del mundo en el que se desenvuelve. Todo esto basado en un correlato neural cuyo sustrato morfofuncional es ahora claro. Por consiguiente, constituye un sinsentido considerar a la holodisnomia como un déficit neurológico y, por antonomasia, un error señalarla como anormal o discapacitada; sus cerebros son diversos y ricos en posibilidades funcionales y operativas, y les permiten procesar información de manera global, dando origen a procesos cognitivo-conductuales que facilitan eficazmente su operación en el mundo, al tiempo que divergen de aquellas expresadas por el fenotipo normativo, sin olvidar que este también es relativo y variable.

\section{REFERENCIAS BIBLIOGRÁFICAS}

Abdullaev, Y., \& Melnichuk, K. (1997). Cognitive operations in the human caudate nucleus. Neuroscience Letters, 151-155.

Adolph, E. (2013). Origins of physiological regulations. Academic Press.

Alexander-Passe, N. (2006). How dyslexic teenagers cope: An investigation of self-esteem, coping and depression. Dyslexia, $12(4), 256-275$.

American Psychiatric Association (APA). (2013). DSM-5: Diagnostic and statistical manual of mental disorders (5th Edition ed.). Washington, DC: American Psychiatric Association.

Bach, S., Brandeis, D., Hofstetter, C., Martin, E., Richardson, U., \& Brem, S. (2010). Early emergence of deviant frontal fMRI activity for phonological processes in poor beginning readers. Neurolmage, 53(2), 682-693.

Barbiero, C., Lonciari, I., Montico, M., Monasta, L., Penge, R., Vio, C., ... Ronfani, L. (2012). The Submerged Dyslexia Iceberg: How Many School Children Are Not Diagnosed? Results from an Italian Study. PLoS ONE, 7(10).

Beaulieu, C., Plewes, C., Paulson, L. A., Roy, D., Snook, L., Concha, L., \& Phillips, L. (2005). Imaging brain connectivity in children with diverse reading ability. Neurolmage, 25(4), 1266-1271.

Bigozzi, L., Tarchi, C., Pinto, G., \& Donfrancesco, R. (2016). Divergent Thinking in Italian Students with and Without Reading Impairments. International Journal of Disability, Development and Education, 63(4), 450-466.

Booth, J. R., Wood, L., Lu, D., Houk, J., \& Bitan, T. (2007). The role of the basal ganglia and cerebellum in language processing. Brain Res., 136-144.

Brown, W., Eliez, S., Menon, V., Rumsey, J., White, C., \& Reiss, A. (2001). Preliminary evidence of widespread morphological variations of the brain in dyslexia. Neurology, 56(6), 781-783.

Burton, M. W. (2001). The role of inferior frontal cortex in phonological processing. Cognitive Science, 25(5), 695-709.

Carroll, J., \& Iles, J. (2006). An assessment of anxiety levels in dyslexic students in higher education. British Journal of Educational Psychology, 76(3), 651-662.

Carroll, J., Maughan, B., Goodman, R., \& Meltzer, H. (2005). Literacy difficulties and psychiatric disorders: Evidence for comorbidity. Journal of Child Psychology and Psychiatry and Allied Disciplines, 46(5), 524-532.

Castles, A., \& Coltheart, M. (1993). Varieties of developmental. Science, 47, 149-180.

Chakravarty, A. (2009). Artistic talent in dyslexia-A hypothesis. Medical Hypotheses, 73(4), 569-571.

Chinn, S. J., \& Crossman, M. (1995). Stress factors in the adolescent. Dyslexia and stress, 49-54. 
Cockcroft, K., \& Hartgill, M. (2004). Focusing on the abilities in learning disabilities: dyslexia and creativity. Education as Change, 61-79.

Cohn, R., \& Neumann, M. (1997). Artistic production in dyslexic children . Neurol. Neurocir. Psiquiatr., 65-69.

Cooper, R. (2009). Neurodiversity and Dyslexia: Compensatory strategies, or different approaches?

Damasio, A., \& Damasio, H. (1983). The anatomic basis of pure alexia. Neurology, 1573-1573.

Dehaene, S. (2014). Reading in the brain revised and extended: response to comments. Mind \& Language, $320-335$.

Dehaene, S., Cohen, L., Sigman, M., \& Vinckier, F. (2005). The neural code for written words: a proposal. Trends in cognitive sciences, 335-341.

Deutsch, G., Dougherty, R., Bammer, R., Siok, W., Gabrieli, J., \& Wandell, B. (2005). Children's reading performance is correlated with white matter structure measured by Diffusion Tensor Imaging. Cortex, 354-363.

Diehl, J., Frost, S., Sherman, G., Mencl, W., Kurian, A., Molfese, P., ... Pugh, K. (2014). Neural correlates of language and nonlanguage visuospatial processing in adolescents with reading disability. Neurolmage, 101, 653-666.

Drake, W. (1968). Clinical and Pathological Findings in a Child with a Developmental Learning Disability. Journal of Learning Disabilities, 1(9), 486-502.

Eckert, M., Berninger, V., Vaden, K., Gebregziabher, M., \& Tsu, L. (2016). Gray Matter Features of Reading Disability: A Combined Meta-Analytic and Direct Analysis Approach. ENeuro, 3(1).

Eide, B., \& Eide, F. (2012). The dyslexic advantage: Unlocking the hidden potential of the dyslexic brain. Penguin.

Eidelberg, D., \& Galaburda, A. (1982). Symmetry and Asymmetry in the Human Posterior Thalamus. Archives of Neurology, 39(6), 325 .

Fiez, J. (2016). Neural Basis of Phonological Short-Term Memory. Neurobiology of Language, 855-862.

Fink, G., Halligan, P., Marshall, J., Frith, C., Frackowiak, R., \& Dolan, R. (1996). Where in the brain does visual attention select the forest and the trees? Nature, 626-628.

Friederici, A. (2016). The Neuroanatomical Pathway Model of Language. Neurobiology of Language, 349-356.

Friederici, A., \& Gierhan, S. (2013). The language network. Current Opinion in Neurobiology, 23(2), $250-254$.

Galaburda, A., Menard, M., \& Rosen, G. (1994). Evidence for aberrant auditory anatomy in developmental dyslexia. Proceedings of the National Academy of Sciences, 91(17), 8010-8013.

Galaburda, A., \& Livingstone, M. (1993). Evidence for a Magnocellular Defect in Developmental Dyslexia. Annals New York Academy of Sciences, 70-82.

Galaburda, A., Sherman, G., Rosen, G., Aboitz, F., \& Geschwind, N. (1985). Developmental Dyslexia: Four consecutive patients with cortical anomaly. Annals of Neurology, 18(2), 222-223.

Galaburda A. (1979). Cytoarchitectonic abnormalities in dyslexia. Annals of Neurology, (6), 94-100.

Geiger, G., \& Lettvin, J. (1987). Peripheral Vision in Persons with Dyslexia. New England Journal of Medicine, $1238-1243$.

Gori, S., \& Facoetti, A. (2014). Perceptual learning as a possible new approach for remediation and prevention of developmental dyslexia. Vision Research.

Goswami, U. (2000). Phonological Representations, Reading Development and Dyslexia: Towards a Cross-Lunguistic Theoretical Framework. Dyslexia, 133-151.

Guttorm, T., Leppänen, P., Poikkeus, A., Eklund, K., Lyytinen, P., \& Lyytinen, H. (2005). Brain event-related potentials (ERPs) measured at birth predict later language development in children with and without familial risk for dyslexia. Cortex, 291-303.

Habib, M., \& Giraud, K. (2013). Dyslexia. En Dulac, M. Lassonde, \& H. B. Sarnat, Handbook of Clinical Neurology (págs. 229235). Marseilles: Elsevier.

Hahn, N., Foxe, J., \& Molholm, S. (2014). Neuroscience and Biobehavioral Reviews Impairments of multisensory integration and cross-sensory learning as pathways to dyslexia. Neuroscience and Biobehavioral Reviews, 47, 384-392.

Hampson, M., Driesen, N., Roth, J., Gore, J., \& Constable, R. (2010). Functional connectivity between task-positive and tasknegative brain areas and its relation to working memory performance. Magnetic Resonance Imaging, 28(8), 10511057.

Hancock, R., Richlan, F., \& Hoeft, F. (2017). Possible roles for fronto-striatal circuits in reading disorder. Neuroscience and Biobehavioral Reviews, 72, 243-260.

Hedenius, M., Persson, J., Alm, P. A., Ullman, M., Howard, J., Howard, D., \& Jennische, M. (2013). Impaired implicit sequence learning in children with developmental dyslexia. Research in Developmental Disabilities, 34(11), 3924-3935.

Heilman, K., \& Acosta, L. (2013). Visual artistic creativity and the brain. Progress in brain research, 19-43.

Hickok, G., \& Poeppel, D. (2004). Dorsal and ventral streams: A framework for understanding aspects of the functional anatomy of language. Cognition, 92(1-2), 67-99.

Hoeft, F., Meyler, A., Hernandez, A., Juel, C., Taylor-Hill, H., Martindale, J., ... Gabrieli, J. (2007). Functional and morphometric brain dissociation between dyslexia and reading ability. Proceedings of the National Academy of Sciences, 104(10), 4234-4239. 
Howard, J., Howard, D., Japikse, K., \& Eden, G. (2006). Dyslexics are impaired on implicit higher-order sequence learning, but not on implicit spatial context learning. Neuropsychologia, 44(7), 1131-1144.

Humphreys, P., Kaufmann, W., \& Galaburda, A., (1990). Developmental dyslexia in women: neuropathological findings in three patients. Annals of Neurology, 28(6), 727-738.

Ingvar, M., Af Trampe, P., Greitz, T., Eriksson, L., Stone-Elander, S., \& Von Euler, C. (2002). Residual differences in language processing in compensated dyslexics revealed in simple word reading tasks. Brain and Language, 83(2), $249-267$.

Jobard, G., Crivello, F., \& Tzourio-Mazoyer, N. (2003). Evaluation of the dual route theory of reading: A meta-analysis of 35 neuroimaging studies. Neurolmage, 20(2), 693-712. https://doi.org/10.1016/S1053-8119(03)00343-4

Kapur, N. (1996). Paradoxical functional facilitation in brain-behavior research. A critical review. Brain, 1775-1790.

Károlyi, C., \& Winner, E. (2004). Dyslexia and visual spatial talents: Are they connected? Students with both gifts and learning disabilities, 95-117.

Kellmeyer, P., Ziegler, W., Peschke, C., Juliane, E., Schnell, S., Baumgaertner, A., ... Saur, D. (2013). Fronto-parietal dorsal and ventral pathways in the context of different linguistic manipulations. Brain and Language, 127(2), 241-250.

Kere, J. (2014). The molecular genetics and neurobiology of developmental dyslexia as model of a complex phenotype. Biochemical and Biophysical Research Communications, 452(2), 236-243.

Kershner, J. (2016). Network dynamics in dyslexia: Review and implications for remediation. Research in Developmental Disabilities, 24-34.

Kiziewicz, M., \& Biggs, I. (2007). CASCADE - Creativity across science, art, dyslexia, education. CASCADE Creativity across Science Art Dyslexia and Education.

Klingberg, T., Hedehus, M., Temple, E., Salz, T., Gabrieli, J., Moseley, M., \& Poldrack, R. (2000). Microstructure of TemporoParietal White Matter as a Basis for Reading Ability. Neuron, 25(2), 493-500.

Klostermann, F., Krugel, L., \& Ehlen, F. (2013). Functional roles of the thalamus for language capacities. Frontiers in Systems Neuroscience, 7 (July), 1-8.

Krishnan, S., Watkins, K., \& Bishop, D. (2016). Neurobiological Basis of Language Learning Difficulties. Trends in Cognitive Sciences, 20(9), 701-714.

Kronbichler, M., Hutzler, F., Staffen, W., Mair, A., Ladurner, G., \& Wimmer, H. (2006). Evidence for a dysfunction of left posterior reading areas in German dyslexic readers. Neuropsychologia, 44(10), 1822-1832.

LeMay, M. (1976). Morphological cerebral asymmetries of modern man, fossil man, and nonhuman primates. Ann NY Acad Sci, 349-366.

Levitin, D., \& Menon, V. (2003). Musical structure is processed in "language" areas of the brain: A possible role for Brodmann Area 47 in temporal coherence. Neurolmage, 20(4), 2142-2152.

Lindsay, G., \& Dockrell, J. (2000). The behaviour and self-esteem of children with specific speech and language difficulties. British Journal of Educational Psychology, 70(4), 583-601.

Linkersdörfer, J., Lonnemann, J., Lindberg, S., Hasselhorn, M., \& Fiebach, C. (2012). Grey matter alterations co-localize with functional abnormalities in developmental dyslexia: An ALE meta-analysis. PLOS ONE, 7(8).

Lozano, A., Ramírez, M., \& Ostrosky-Solis, F. (2003). The neurobiology of developmental dyslexia: a survey. Revista de neurología, 1077-1082.

Maisog, J., Einbinder, E., Flowers, D., Turkeltaub, P., \& Eden, G. (2008). A meta-analysis of functional neuroimaging studies of dyslexia. Annals of the New York Academy of Sciences, 1145, 237-259.

Martínez-Méndez, R., Martínez-Martínez, E., Villafán-Monroy, H., Guzmán-López, J., Fuentes-Farías, A., Romo-González, T., ... Gutierrez-Ospina, G. (2013). 4. Body and brain plasticity: Unraveling its principles through blindness. Research Signpost India Recent Res. Devel. Neurosci, 37661(4), 89-107.

Maughan, B., \& Carroll, J. (2006). Literacy and mental disorders. [Miscellaneous]. Current Opinion in Psychiatry, 19(4), 350354 .

McCandliss, B., Cohen, L., \& Dehaene, S. (2003). The visual word form area: Expertise for reading in the fusiform gyrus. Trends in Cognitive Sciences, 7(7), 293-299.

McNorgan, C., Alvarez, A., Bhullar, A., Gayda, J., \& Booth, J. (2011). Prediction of reading skill several years later depends on age and brain region: Implications for developmental models of reading. $J$ Neurosci, 9641-9648.

Meyler, A., Keller, T., Cherkassky, V., Gabrieli, J., \& Just, M. (2008). Modifying the Brain Activation of Poor Readers during Sentence Comprehension with Extended Remedial Instruction: A Longitudinal Study of Neuroplasticity. Neuropsychologia, 2580-2592.

Moro, A., Tettamanti, M., Perani, D., Donati, C., Cappa, S., \& Fazio, F. (2001). Syntax and the brain: Disentangling grammar by selective anomalies. Neuroimage, 110-118.

Niogi, S., \& McCandliss, B. (2006). Left lateralized white matter microstructure accounts for individual differences in reading ability and disability. Neuropsychologia, 44(11), 2178-2188. 
Olagboyega, K. (2008). The effects of dyslexia on language acquisition and development. Scientific and Technical Reports of Faculty of Engineering and Resourse Science, Akita University, 23-27.

Paulesu, E, Demonet, J., Fazio, F., McCrory, E., Chanoine, V., Brunswick, N., ... Frith, U. (2001). Dyslexia: cultural diversity and biological unity. Science, 291, 2165-2168.

Paulesu, E., Frith, C., \& Frackowiak, R. (1993). The neural correlates of the verbal component of working memory. Nature, 342-345.

Pearson, R. (2017). Dislexia: una forma diferente de leer. Buenos Aires: Paidós.

Pennington, B. (2006). From single to multiple deficit models of developmental disorders. Cognition, 101(2), 385-413.

Perrachione, T., Del Tufo, S., \& Gabrieli, J. (2011). Human voice recognition depends on language ability. Science, 333(6042), 595.

Perrone-Bertolotti, M., Kauffmann, L., Pichat, C., Vidal, J., \& Baciu, M. (2017). Effective Connectivity between Ventral Occipito-Temporal and Ventral Inferior Frontal Cortex during Lexico-Semantic Processing. A Dynamic Causal Modeling Study. Frontiers in Human Neuroscience, 11(June), 1-13.

Peterson, R., Pennington, B., Olson, R., \& Wadsworth, S. (2014). Longitudinal Stability of Phonological and Surface Subtypes of Developmental Dyslexia. Sci Stud Read., 347-362.

Peterson, R., \& Pennington, B. (2012). Seminar: Developmental Dyslexia. National Institute of Health, 379(9830), $1997-2007$.

Poldrack, R., Wagner, A., Prull, M., Desmond, J., Glover, G., \& Gabrieli, J. (1999). Functional Specialization for Semantic and Phonological Processing in the Left Inferior Prefrontal Cortex. Neuroimage, 15-35.

Price, C. (2012). A review and synthesis of the first 2oyears of PET and fMRI studies of heard speech, spoken language and reading. Neurolmage, 62(2), 816-847.

Pugh, K., Mencl, W., Jenner, A., Katz, L., Frost, S., Lee, J., . . . Shaywitz, B. (2000). Functional Neuroimaging Studies of Reading and Reading Disability (Developmental Dyslexia). Research Reviews, 207-213.

Rack, L. (1981). Developmental dyslexia and literary creativity: Creativity in the area of deficit. Journal of Learning Disabilities, 14(5), 262-263.

Ramus, F., Altarelli, I., Jednoróg, K., Zhao, J., \& Scotto di Covella, L. (2018). Neuroanatomy of developmental dyslexia: Pitfalls and promise. Neuroscience and Biobehavioral Reviews, 84(August 2017), 434-452.

Rawson, M. (1978). Dyslexia and learning dissabilities: Their relationship. Bulletin of The Orton Society, 43-61.

Richlan, F. (2014). Functional neuroanatomy of developmental dyslexia: the role of orthographic depth. Frontiers in Human Neuroscience, 8(May), 1-13.

Richlan, F., Kronbichler, M., \& Wimmer, H. (2009). Functional abnormalities in the dyslexic brain: A quantitative metaanalysis of neuroimaging studies. Human Brain Mapping, 30(10), 3299-3308.

Richlan, F., Kronbichler, M., \& Wimmer, H. (2011). Meta-analyzing brain dysfunctions in dyslexic children and adults. Neurolmage, 56(3), 1735-1742.

Richlan, F., Kronbichler, M., \& Wimmer, H. (2013). Structural abnormalities in the dyslexic brain: A meta-analysis of voxelbased morphometry studies. Human Brain Mapping, 34(11), 3055-3065.

Riddick, B., Sterling, C., Farmer, M., \& Morgan, S. (1999). Self-esteem and anxiety in the educational histories of adult dyslexic students. Dyslexia, 5(4), 227-248.

Saur, D., Kreher, B., Schnell, S., Kummerer, D., Kellmeyer, P., Vry, M., ... Weiller, C. (2008). Ventral and dorsal pathways for language. Proceedings of the National Academy of Sciences, 105(46), 18035-18040.

Schneps, M. (2015). The advantages of dyslexia. Scientific American Mind, 24-25.

Schneps, M., Brockmole, J., Rose, L., Pomplun, M., Sonnert, G., \& Greenhill, L. (2011). Dyslexia linked to visual strengths useful in astronomy. Bulletin of the American Astronomical Society.

Schneps, M., Rose, L., \& Fischer, K.. (2007). Visual Learning and the Brain: Implications for Dyslexia. Mind, Brain, and Education, 1(3), 128-139.

Seghier, M., Neufeld, N., Zeidman, P., Leff, A., Mechelli, A., Nagendran, A., ... Price, C. (2012). Reading without the left ventral occipito-temporal cortex. Neuropsychologia, 50(14), 3621-3635.

Shaywitz, B., Shaywitz, S., Pugh, K., Mencl, W., Fulbright, R., Studlarski, P., \& Gore, J. (2002). Disruption of posterior brain systems for reading in children with developmental dyslexia. BiologicalPsychiatry, 101-110.

Shaywitz, S., \& Shaywitz, B. (2008). Paying attention to reading: The neurobiology of reading and dyslexia. Development and Psychopathology, 20(4), 1329-1349.

Shaywitz, S., Shaywitz, B., Fulbright, R., Skudlarski, P., Mencl, W., Constable, R., . . . Gore, J. (2003). Neural Systems for Compensation and Persistence: Young Adult Outcome of Childhood Reading Disability. Society of Biological Psychiatry, 25-33.

Shaywitz, S., Escobar, M., Shaywitz, B., Fletcher, J., \& Makuch, R. (1992). Evidence that dyslexia may represent the lower tail of a normal distribution of reading ability. The New England Journal of Medicine, 145-150. 
Silani, G., Frith, U., Demonet, J., Fazio, F., Perani, D., Price, C., ... Paulesu, E. (2005). Brain abnormalities underlying altered activation in dyslexia: A voxel based morphometry study. Brain, 128(10), 2453-2461.

Simos, P., Fletcher, J., Sarkari, S., Billingsley, R., Denton, C., \& Papanicolaou, A. (2007). Altering the Brain Circuits for Reading Through Intervention: A Magnetic Source Imaging Study. Neuropsychology, 21(4), 485-496.

Squire, L., Berg, D., Bloom, F., Du Lac, S., Ghosh, A., \& Spitzer, N. (2012). Fundamental neuroscience. Academic Press.

Stein, J. (2001). The Magnocellular Theory of Developmental Dyslexia. Dyslexia, 7(1), 12-36.

Stein, J. (2018). Author's Accepted Manuscript Developmental dyslexia. Neuropsychologia.

Steinbrink, C., Ackermann, H., Lachmann, T., \& Riecker, A. (2009). Contribution of the anterior insula to temporal auditory processing deficits in developmental dyslexia. Human Brain Mapping, 30(8), 2401-2411.

Swanson, H. (1984). Semantic and visual memory codes in learning disabled readers. Journal of Experimental Child Psychology, 124-140.

Swick, D., Ashley, V., \& Turken, A. (2008). Left inferior frontal gyrus is critical for response inhibition. BMC Neuroscience, 9, 111.

Temple, E., Deutsch, G., Poldrack, R., Miller, S., Tallal, P., Merzenich, M., \& Gabrieli, J. (2003). Neural deficits in children with dyslexia ameliorated by behavioral remediation: Evidence from functional MRI. Proceedings of the National Academy of Sciences, 100(5), 2860-2865.

Tettamanti, M., Buccino, G., Saccuman, M., Gallese, V., Danna, S., \& Scifo, P. (2005). Listening to action-related sentences activates fronto-parietal motor circuits. Journal of Cognitive Neuroscience, 273-281.

Turken, A., \& Dronkers, N. (2011). The Neural Architecture of the Language Comprehension Network: Converging Evidence from Lesion and Connectivity Analyses. Frontiers in System Neuroscience, 5(February), 1-20.

Uddin, L., Nomi, J., Hebert-Seropian, B., Ghaziri, J., \& Boucher, O. (2017). Structure and function of the human insula. J Clin Neurophysiol., 300-306.

Vandermosten, M., Boets, B., Poelmans, H., Sunaert, S., Wouters, J., \& Ghesquière, P. (2012). A tractography study in dyslexia: Neuroanatomic correlates of orthographic, phonological and speech processing. Brain, 135(3), 935-948.

Vlachos, F., Papathanasiou, I., \& Andreou, G. (2007). Cerebellum and reading. Folia Phoniatrica et Logopaedica, 59(4), 177183.

Von, K. (2001). Visual-spatial strength in dyslexia: Rapid discrimination of impossible figures. Journal of Learning Disabilities, 34(4), 380-391.

Von Károlyi, C., Winner, E., Gray, W., \& Sherman, G. (2003). Dyslexia linked to talent: Global visual-spatial ability. Brain and Language, 85(3), 427-431.

Watkins, N., Vargha-khadem, F., Ashburner, J., Passinham, R., Connelly, A., ... Gadian, D. (2002). MRI analysis of an inherited speech and language disorder: Structural brain abnormalities. Brain, 125, 465-478.

Wimmer, H., \& Schurz, M. (2010). Dyslexia in Regular Orthographies: Manifestation and Causation. Dyslexia, $283-299$.

Wimmer, H., Schurz, M., Sturm, D., Richlan, F., Klackl, J., Kronbichler, M., \& Ladurner, G. (2010). A dual-route perspective on poor reading in a regular orthography: An fMRI study. Cortex, 46(10), 1284-1298.

Yeatman, J., Dougherty, R., Ben-Shachar, M., \& Wandell, B. (2012). Develooment of white matter and reading skills. Proceedings of the National Academy of Sciences, 1-9.

Zakopoulou, V., Anagnostopoulou, A., Christodoulides, P., Stavrou, L., Sarri, I., Mavreas, V., \& Tzoufi, M. (2011). An interpretative model of early indicators of specific developmental dyslexia in preschool age: A comparative presentation of three studies in Greece. Research in Developmental Disabilities, 32(6), 3003-3016.

Zoccolotti, P., De Luca, M., Di Pace, E., Gasperini, F., Judica, A., \& Spinelli, D. (2005). Word length effect in early reading and in developmental dyslexia. Brain and Language, 93(3), 369-373. 